DOI: https://doi.org/10.46296/yc.v5i8edespen.0089

\title{
EMPOWERMENT COMO HERRAMIENTA DE GESTIÓN ESTRATÉGICA EFECTIVA EN EL DESEMPEÑO LABORAL EN EQUIPOS DE TRABAJO
}

\section{EMPOWERMENT AS AN EFFECTIVE STRATEGIC MANAGEMENT TOOL IN WORK PERFORMANCE IN WORK TEAMS}

\author{
Piguave-Bello Miryam Margarita ${ }^{1 *}$; Vegas-Meléndez Hilarión José ${ }^{2}$ \\ ${ }^{1}$ Estudiante de Maestría en Administración de Empresas, Pontificia Universidad \\ Católica del Ecuador, sede Manabí. Portoviejo - Ecuador. \\ ${ }^{1}$ Docente e investigador de pre y posgrado de la Pontificia Universidad Católica del \\ Ecuador, sede Manabí. Portoviejo - Ecuador.
}

*Correo: mpiguave0346@pucem.edu.ec

\begin{abstract}
Resumen
El presente trabajo investigativo, tiene como objetivo principal establecer el significado que tiene el empowerment como herramienta de gestión estratégica de los equipos de trabajo en el desempeño laboral en las empresas públicas, la cual tiene como referencia de estudio a la Empresa de Producción y Desarrollo Estratégico (EPULEAM). Dada las características de la temática y objetivos propuestos en la investigación, la misma se diseñó desde la perspectiva metodológica cualitativa y de método deductivo, con un nivel descriptivo y de tipo documental, desarrollada desde la investigación de campo en la que se contó con la contribución de destacados actores dentro de la EPULEAM. El instrumento de recolección de información se basó en la observación directa y la entrevista en profundidad aplicada a directivos de esta institución, permitiendo proporcionar información relevante para la posterior construcción de los datos que permitieron dar como resultados categorías y descriptores, soportados por un análisis de tipo hermenéutico; para esto se utilizaron pasos propuestos desde la denominada Teoría Fundamentada: descripción analítica, ordenación conceptual, triangulación teórica y el proceso de codificación (abierta, axial y selectiva); entre las conclusiones relevantes destaca el hecho de que el empowerment es una herramienta de gestión viable para todo tipo de empresa (públicas y privadas), en la que se viabilizan espacios para la generación de nuevas oportunidades de negocio para la organización empresarial.
\end{abstract}

Palabras claves: Empowerment, equipos de trabajo, gestión estratégica, Teoría Fundamentada.

\begin{abstract}
The main objective of this investigative work is to establish the meaning of empowerment as a strategic management tool for work teams in job performance in public companies, which has as a study reference the Strategic Production and Development Company (EPULEAM). Given the characteristics of the theme and objectives proposed in the research, it was designed from the qualitative methodological perspective and deductive method, with a descriptive and documentary level, developed from the field research in which the contribution was made. of prominent actors within EPULEAM. The information collection instrument was based on direct observation and an in-depth interview applied to directors of this institution, allowing to provide relevant information for the subsequent construction of the data that allowed categories and descriptors to be given as results, supported by an analysis of the type hermeneutical; For this, steps proposed from the so-called Grounded Theory were used: analytical description, conceptual ordering, theoretical triangulation and the coding process (open, axial and selective); Among the relevant conclusions, the fact that empowerment is a viable management tool for all types of companies (public and private) stands out, in which spaces are made possible to generate new business opportunities for the business organization.
\end{abstract}

Keywords: Empowerment, work teams, strategic management, Grounded Theory.

Información del manuscrito:

Fecha de recepción: 16 de noviembre de 2020.

Fecha de aceptación: 08 de enero de 2021.

Fecha de publicación: 29 de enero de 2021. 


\section{Introducción}

La actualidad que plantea el siglo XXI implica estar inmerso en un mundo globalizado en todos los órdenes de la sociedad, esto a su vez conmina hacia la búsqueda del desarrollo de estrategias que permitan el perfeccionamiento y la optimización de los procedimientos administrativos; esto sustentado en la cultura organizacional de una empresa, además de garantizar la productividad y el rendimiento profesional del talento humano. Para ello, es necesario considerar los múltiples aspectos que se encuentran presente en el marco de una empresa que se considere competitiva, por eso es necesario desarrollar estrategias de crecimiento y desarrollo, especialmente dirigidas a la potenciación del capital humano adscrito a la empresa.

En América Latina el ámbito de la gerencia y la gestión del talento humano se encuentra inmerso por cambios y transformaciones, en cuyo contexto, el personal directivo y de gerencia de empresas debe diseñar estrategias, que le posibiliten alcanzar las metas a nivel organizacional, de manera de coadyuvar a que los trabajadores sientan la valoración de su trabajo, en el desempeño de sus funciones; en pro de combatir enfoques anteriores en los que no se consideraban las opiniones $y$ participación del personal en temas esenciales para el crecimiento de la empresa. A fin de contextualizar aún mejor la idea, Chiavola, et al. (2008) consideran que el nivel gerencial de mayor jerarquía en las empresas existía una tendencia de alejamiento en la que:

No se relacionaba
adecuadamente con sus
subordinados, ejerciendo un
liderazgo fundamentado en el
poder y primordialmente con un
estilo coercitivo sin darle
oportunidad o posibilidades de
participar en la toma de
decisiones, creando situaciones
de desmotivación y poco
rendimiento laboral. (pág.131)

De allí, que sea preciso considerar como enfoque alterno para combatir toda esta situación, el uso del empowerment como parte de la gestión estratégica, lo cual se puede asumir desde una concepción de 
potenciación y empoderamiento, desde cuya sustentación se debe delegar responsabilidades a los subordinados que ostenten cargos directivos y administrativos, lo que implica delegar en ellos, cierto nivel de autonomía, autoridad y poder de decisión ante temas relevantes, en pro de lograr la excelencia y la efectividad. Esto, dado que coincidimos nuevamente con el enfoque de Chiavola, et al. (2008), quienes alegan que el empoderamiento "permite que los empleados se sientan cómodos y tengan sentido de orientación, posesión y responsabilidad para poder adquirir las destrezas requeridas para el puesto. Así mismo, se sienten capacitados, haciéndolos acreedores de conocimientos y estímulos para tomar sus decisiones" (p.132).

El empoderamiento posibilita que los colaboradores tengan sentido de pertenencia y orientación de hacia dónde deben conducirse; y que se sientan responsables de demostrar sus habilidades y competencias en correspondencia con las funciones de desempeño de cada uno de sus cargos. De igual manera, este enfoque permite mayor seguridad e independencia lo que a su vez refuerza sus conocimientos $y$ destrezas, aumentando su capacidad resolutiva frente a la toma oportuna de decisiones.

Aunado a lo anterior, asignar mayores responsabilidades en los trabajadores les proporciona confianza y compromiso con la organización y les concede la posibilidad de sentir que están apoderados de su cargo, de su trabajo, dejando de lado las estructuras piramidales, desde las cuales, la toma de decisiones se hace sólo a nivel de la alta gerencia $\mathrm{y}$ en los mayores niveles de la organización, lo que podría originar nuevos beneficios para la organización.

Asimismo, el empoderamiento eleva el nivel de satisfacción, autoestima y autonomía de las personas que forman parte del talento humano de la empresa, debido al aumento progresivo de la iniciativa, creatividad, sentido de pertenencia, fomentando la cultura del empoderamiento que les identifica dentro de la empresa,minimizando considerablemente la resistencia al cambio; además de aquello, se optimiza la interacción y las 
relaciones interpersonales que se promueve entre los compañeros, y se crea una notable motivación y una actitud positiva ante los retos que se presentan en el ámbito laboral. De allí que, se considere que, si las empresas adoptaran esta herramienta de gestión organizacional, los trabajadores se sentirían aún más valorados, fortaleciendo su sentido de pertenencia y compromiso con la organización.

Desde este marco referencial, es consideración de los investigadores, abordar el estudio de este tema desde una perspectiva holística cuyo objetivo principal es interpretar el significado del empowerment como herramienta de gestión estratégica en el desempeño laboral desarrollado a través de equipos de trabajo en la Empresa Pública ULEAM, en el área administrativa; todo ello, buscando su significación de la perspectiva gerencial. Esto último obedece al hecho de que se presume la necesidad de incluir dentro de su filosofía de gestión empresarial el empoderamiento para potenciar el compromiso y la motivación por parte de quienes allí laboran, lo cual puede inducir en un desempeño cada vez autónomo, innovador y generador de cambios organizacionales. Por lo expuesto anteriormente, se considera importante abordar esta temática, a partir de las siguientes interrogantes: ¿Qué significado tiene el empowerment como herramienta de gestión estratégica de los equipos de trabajo en el desempeño laboral?

La investigación se plantea como objetivo principal establecer el significado que tiene el empowerment como herramienta de gestión estratégica de los equipos de trabajo en el desempeño laboral en las empresas públicas, es especial a la EPULEAM teniendo como acompañantes objetivos específicos como: identificar las estrategias de gestión en equipos de trabajo y; describir el empowerment como generador de nuevas habilidades en equipos de trabajo frente a las oportunidades de negocio en las empresas públicas. Se desarrolló bajo el direccionamiento de la línea de investigación: Administración y la gestión de la calidad; representado una temática de creciente interés ante la comunidad científica involucrada en este tópico de la gerencia y la gestión estratégica, 
dado que, ésta se concibe como una herramienta que permitirá generar un nuevo constructo organizacional en la Empresa Pública ULEAM, cuyo horizonte es la de que su personal se sienta aún más motivados y comprometidos con la cultura de la empresa, reforzando así su comportamiento organizacional; se busca que la metódica pueda generar cambios significativos en temas como la comunicación, autonomía, trabajo en equipo, potenciación de las habilidades y destrezas, valorando el trabajo razonado de los equipos de trabajo.

La investigación permite ofrecer aportes epistemológicos y nuevos enfoques, así como de resultados que conduzcan al perfeccionamiento de una gestión sustentada en el empowerment como herramienta estratégica de la gerencia para el óptimo desempeño laboral en la Empresa Pública ULEAM y poder aportar información de interés en aras de coadyuvar hacia la solución de la situación problemática que permite el punto de partida de este trabajo de investigación.

\section{Empowerment: herramienta de gestión estratégica desde un constructo teórico}

En las ciencias administrativas el desempeño laboral efectivo es una de las fortalezas más importantes de las que debe disponer toda organización, dado que la productividad, los logros y alcances que se obtengan dependen en buena medida del desempeño laboral del personal directivo y demás trabajadores de una institución determinada, lo cual podría concretarse, toda vez que se asuma el empowerment como herramienta de gestión estratégica.

Por ello, el abordaje temático acerca del empowerment como herramienta de gestión estratégica ha adquirido creciente interés en el mundo globalizado hoy en día, tanto en el área académica como en el contexto empresarial, como una dimensión esencial en el estudio de lo empresarial, que permite la búsqueda de la permanencia exitosa en el mundo del mercado actual, tan dinámico y cambiante. Uno de sus principales teóricos, Rappaport (1984) define el empowerment como "el proceso por el cual, las personas, organizaciones y comunidades 
adquieren dominio y control de sus vidas. Este modelo intentaba mejorar la calidad mediante la potencialización de recursos individuales, colectivos y comunitarios" ( $p, 32)$; este aporte de Rappaport (1984) reafirma lo expuesto por anteriores referentes en este trabajo investigativo, en la que se destaca la posibilidad de ser autonómos en las actividades que cada individuo desarrolla, y que, si es posible trasladar tal condición de autonomía al ámbito laboral, en la que se puede encontrar resultados muy favorables para la organización.

Con el pasar del tiempo ha adquirido mayor protagonismo la concepción del empowerment como proceso en sucesivos contextos que han beneficiado el capital humano de cualquier organización, en este sentido, las organización en todo el mundo empezaron a reemplazar su estructura tradicional por un mayor compromiso del personal, ya que, la estructura tradicional funcionaba de forma piramidal, en donde las funciones, supervisiones $y$ decisiones eran tomadas desde la punta de la pirámide, para asegurar que el trabajo fuese más rápido y consistente. Para (Porret, 2014, p.104), señala que "han de desaparecer los excesivos niveles jerárquicos en los cuales existen sólo puestos de trabajo", para ello, la estructura de la organización debe verse en forma de circulo o de red, involucrando a todos los grupos o equipos coordinados trabajando en función de un mismo objetivo.

Con este nuevo enfoque se le da la oportunidad al colaborador de dar lo mejor de sí, obteniendo de ellos un mayor rendimiento en el plano humano y profesional, reemplazando el modelo jerárquico de impartir órdenes, ejercido desde el nivel más alto de la empresa, e impulsando de forma estratégica el trabajo en equipo.

Asimismo, para Wilson (2000) "El empowerment organizacional puede ir del control de los trabajadores de toda la organización al hecho que los individuos tengan voz y voto en la manera en que funcionan en sus respectivos trabajos" (p.16). Por otro lado, "El empowerment consiste en la liberación de los conocimientos, la experiencia y la capacidad de motivarse que tienen las personas" (Cucchi, 2008, p.60). Es decir, el empowerment centra su atención en la gestión estrategia ayudando a 
mejorar la eficiencia en la organización y en las actividades esenciales como lo es la comunicación corporativa y gestión de talento humano y además de estudiar aquellos factores externos e internos que pueden influir en su aplicación efectiva, ya que si desde la gerencia se pone en práctica esta nueva filosofía empresarial, el líder de la organización deberá delegar poder y autoridad a sus colaboradores, y de esta manera, la toma de decisiones ya no depende de una sola persona, sino de un colectivo, que podrán llevar a cabo su trabajo con autoridad, compromiso y responsabilidad propia.

De igual manera, (Ivancevich et al. 2006) expone que el empowerment, empoderamiento o facultamiento "Es alentar y ayudar a individuos y grupos para que tomen decisiones que afectan su entorno laboral" (p.394). El empowerment, no es más que la facultad de ceder la autoridad, el poder 0 autoridad a las personas que necesitan tomar decisiones y participar activamente en la organización, dándoles la oportunidad de ser los protagonistas de los buenos resultados. Razón por la cual, la gerencia debe proporcionar a los empleados toda la información, conocimientos y recursos requeridos para desempeñar las tareas asignadas, ya que de esta manera permite que la ejecuten con miras de lograr los resultados deseados, siempre y cuando se ajusten a la misión y visión institucional.

Por otra parte, el empowerment en el proceso de avance del talento humano dentro de una organización, representa una herramienta estratégica importante para el desarrollo de las habilidades y destrezas de los colaboradores, el lograr el compromiso de los trabajadores de una organización con esta nueva filosofía pretende de un cambio en las actitudes de la gerencia, la cual debe estar enfocada en dirigir al personal a su cargo hacia la construcción de un lugar de trabajo en donde el empowerment sea la base de la cultura de la organización, de toda la gerencia.

Sin duda alguna, para que la implementación del empowerment, pueda tener éxito en una organización se necesita de un liderazgo que sea eficaz capaz de 
dirigir a los colaboradores hacia el rumbo deseado, deben hacer que estos se identifiquen con los valores y la misión de la empresa, permitiendo que puedan aportar ideas para la ejecución de los objetivos trazados.

Desde estas perspectivas, se concreta la necesidad de disponer de un líder emprendedor dentro de las empresas, que se sustente en el empowerment, como estrategia básica de desarrollo del desempeño laboral, que planifique acciones de gestión en correspondencia con las competencias de los profesionales que conforman el talento humano, como capital intangible básico para el éxito empresarial. En ese orden, en un trabajo de investigación divulgado por la revista FORBES (2017) que se denominó: "Cómo convertirte en emprendedor desde la oficina" se hace alusión a la relevancia de valorar el trabajo del personal de las organizaciones, considerando para ello cuatro aristas esenciales a saber: "autonomía, confianza, responsabilidad y retos laborales". (FORBES, 2017, en Amezcua, Pérez y Quiroz, 2019). Además, este líder debe de poseer características muy importantes y particulares, donde el mismo sea capaz de incentivar a sus empleaos para que se incorporen a sus labores con un estado de ánimo más alegre y cordial.

En el marco de las ideas anteriores, se asumen los enfoques de (Chiavenato, 2009) sobre lo que se debe adoptar en todo proceso de gestión: autoridad, información, recompensas, competencias, dentro de lo cual, el líder de la organización puede delegar autoridad a algunos de los miembros de la gestión directiva, donde todos deben conocer y manejar la información de lo que están desarrollando cada uno de los miembros del equipo, mismos que deben sentirse satisfechos, con respecto a sus incentivos laborales y desplegar sus funciones en correspondencia con sus destrezas y habilidades profesionales. De allí que coincidamos con los autores que adoptan la postura de que el empowerment o empoderamiento se configura en una herramienta que ofrece la posibilidad de que el trabajador de la empresa adquiera el poder para auto direccionar su labor y procure el aseguramiento de los recursos requeridos, para llevar a cabo su desempeño según los 
mejores estándares de calidad y productividad, lo cual, en palabras de (Amezcua, et al, 2019), se refiere a que la tendencia de hoy día radica en el desarrollo de una gestión empresarial basada en el uso del empowerment, lo cual expresan de la siguiente forma:

En la actualidad, las tendencias laborales incitan a los líderes de las empresas para que hagan uso del empowerment, ya que es indispensable desarrollar una mayor autonomía y autogestión en los procedimientos, es por ello, que esta herramienta aparece como un elemento que permite guiar a las personas hacia caminos en los cuales sean especialistas y tomen decisiones eficientemente. Dentro de las empresas, se requiere que haya un liderazgo que guíe el camino de las personas y se encuentre en una constante búsqueda de actualizaciones de competencias para gestionar los procedimientos que la persona desarrolla. (p. 34).

Al derivar la respectiva deducción sobre lo propuesto por los autores en la cita anterior, puede decirse que, el empowerment está considerado como un sustento estratégico, que implica la búsqueda de la eficiencia y la efectividad, desde el ámbito empresarial, donde es preciso el ejercicio de una gestión que se conduzca al desarrollo de habilidades para la gestión efectiva de los mecanismos administrativos productivos, efectuados por el talento humano de la empresa.

\section{Empowerment como estrategia para equipos de trabajo. Espacio para la discusión}

Desde este espacio se procura abrir una discusión que bien permita establecer pautas de seguimiento por parte de aquella gerencia que bien considera necesaria la instrumentación de esta herramienta gerencial, la cual busca que los equipos de trabajo trabajen con metas y objetivos estratégicos formulados desde la filosofía empresarial. La investigación procura una discusión basada en la realidad empresarial; para ello, se asume analizar la propuesta del empowement desde el comportamiento organizacional de la Empresa Pública ULEAM, cuya estructura está dada para que esta herramienta de trabajo pueda ser adecuadamente ejecutada. Recordando la interrogante planteada al inicio de este articulo: 
¿Qué significado tiene el empowerment como herramienta de gestión estratégica de los equipos de trabajo en el desempeño laboral?, se genera un razonamiento entre lo teórico y la praxis en las empresas públicas.

No cabe duda que las estrategias innovadoras vinculadas al empowerment permiten asumir una concepción de potenciación y empoderamiento por parte de los equipos de trabajo en cualquier tipo de organización, donde la experiencia gerencial juega un papel determinante; en ese sentido Franco (2021), considera que "es importante que se alcancen los objetivos y metas establecidas, se espera que el trabajador presente soluciones y no problemas" (p.1); por su parte, Pincay (2021) asegura que el al empowerment representa para el trabajador el "sentirse dueño de la empresa, gracias a ese poder que le otorga el empleador" (p.3).

Por otro lado, se parte de la concepción de que el empowerment genera nuevas habilidades en los equipos de trabajo, lo cual redunde directamente en su desempeño laboral, garantizando un mayor nivel de alcance de las metas establecidas. Franco (2021), asume que "esas habilidades se van perfeccionando en la medida que el trabajador logra entender la responsabilidad institucional que tiene, además obtener la autoridad y autonomía en el área de su competencia" (p.2); Pincay (2021), asume que "se aprende a trabajar en equipo, se siente con el poder de ayudar en la decisión; hay aprendizaje continuo y, eso es una habilidad" (p.3)

Otro aspecto resaltante sobre el rol del empowerment en las organizaciones es el de la posibilidad de fortalecer el liderazgo empresarial frente a las oportunidades de negocio; ante ello Franco (2021) cree prudente "organizar 0 reorganizar los procesos que se vinculen con la idea de negocios, el cual permita un crecimiento individual e institucional", en este sentido Pincay (2021) resalta la "confianza en el impacto de un buen liderazgo, donde la valoración del trabajador es de suma importancia; la innovación puede ser parte del resultado de un buen liderazgo" (p.4)

De acuerdo con las entrevistas realizadas a funcionarios, los cuales representan puestos importantes en 
EPULEAM el contraste de este análisis se entiende que el empowerment es una herramienta estratégica que denota un sentido de responsabilidad y libertad a sus empleados en la toma de decisiones. Para asociar esa acción es fundamental tomar en consideración su desenvolvimiento, la producción intangible del recurso humano; con aportes de ideas y sobre todo el compromiso para asumir competencias específicas que se le encomendaren por mandos superiores sobre funciones a ejecutar.

Dentro del sector público, y más aún en empresas públicas, la instrumentación de herramientas gerenciales como el empowerment es algo difícil su aplicabilidad, esto de acuerdo a sus parámetros normativos -rígidos en la administración pública- ya que los niveles de organización se sujetan a estructuras jerárquicas y de rendición de cuentas directa ante los órganos superiores, fiscalización y control; es decir, no se pueden tomar decisiones de manera libre y voluntaria; toda idea y acción aun cuando fuese favorable para la empresa pública en la que incluso este orientada a darle amplitud y mejoramiento de la calidad en la gestión del servicio en la institución, estas deben ser consultadas a sus altos mandos jerárquicos.

Ante estos hechos en relación con las observancias y a manera de contraste, el empowerment es utilizado como una propuesta estratégica e innovadora para ambos sectores, aunque sea muy claro que su aprovechamiento es de mayor utilización en la gestión privada. Sin embargo, desde la perspectiva gerencial de la EPULEAM, se observa claras señales de un interés en aplicar el empowerment dada sus significativas ventajas que bien permiten direccionar procesos en la que los empleados se sienten parte de las soluciones, en la que los equipos de trabajo dan cumplimiento a los objetivos y metas que se establecen, los jefes departamentales establecen libertad organizativa y autonomía responsable a los trabajadores para resolver desde su perspectiva y experiencia, con ello se busca generar compromiso y motivación en el cumplimiento de los fines estratégicos de la empresa pública ULEAM. 


\section{Acercamiento metodológico}

El enfoque cualitativo es asumido desde la perspectiva investigativa, vinculándose al nivel descriptivo crítico y de carácter inductiva, además de la observación directa y analítica; estableciendo una rigurosidad en la saturación teórica en relación a las principales definiciones 0 posturas sobre el empowement y sus principales características como herramienta de gestión estratégica en organizaciones altamente efectivas y con resultados en la mira; por otro lado, se asume la entrevista en profundidad como instrumento de recolección de información primaria, permitiendo identificar la postura de quienes tienen la responsabilidad de gerenciar las decisiones, así como las de quienes reciben las instrucciones para su aplicabilidad y ejecución. La entrevista en profundidad permite:

Obtener las concepciones personales de los entrevistados sobre la situación objeto de la investigación en medio de un esfuerzo de inmersión y reinversión con intimidad y familiaridad para sinceramente reconstruir a través de la discursividad el punto de vista (perspectiva) del informante (o actor relevante) en el cual se ubica la investigación y su contexto (Vegas M., 2016, p. 421)

Los elementos 0 hallazgos resultantes emanados desde la entrevista en profundidad permiten establecer bifurcaciones sobre las ideas planteadas en la investigación. La posterior triangulación entre las teorías revisadas, la postura de los entrevistados y la observación critico analítica de los investigadores permitirá encontrar espacio para la disertación académica y científica sobre la temática del empowerment y su aplicabilidad estratégica en el contexto de las empresas públicas, especialmente a la EPULEAM, lo cual representa una oportunidad para elevar sus niveles de eficiencia laboral, así como de sus oportunidades para la visión de nuevos negocios.

El método hermenéutico es el utilizado para el análisis de los resultados y hallazgos, partiendo desde el problema como aspecto empírico a considerar. Luego se considera el aporte dado por los entrevistados a fin de poder generar los correspondientes "códigos in 
vivo" que dan paso a las correspondientes categorías, y estas a su vez, a los descriptores gerenciales que forman parte de las propuesta de la presente investigación; en este constructo se toma en consideración parte del procedimiento de categorización propuesto en la Teoría Fundamentada (Strauss y Corbin, 2016) en la que "se define como establecer las categorías para luego alcanzar la denominada teoría sustantiva" (p.12). Esta propuesta de la Teoría Fundamentada permite no solo alcanzar una teoría sustantiva, sino que también conlleva a la formulación de la teoría formal de una investigación; sin embargo, para los efectos de esta investigación solo se asume hasta el proceso de categorización lo cual contribuye en la generación de variables importantes a considerar por las organizaciones, en especial la Empresa Pública ULEAM.

Las categorías resultantes del análisis hermenéutico de los investigadores representaron un ejercicio de habilidad comprensiva e interpretativa en la que se tomaron en cuenta todas las variables presentes en la investigación:
Teorías representativas (saturación), discurso de actores informantes (conocimiento empírico y formal), así como la acción sistemática y creativa -al mismo tiempo- de los propios investigadores; esto conllevo a establecer descriptores que buscan mostrar el significado de la temática del empowerment como herramienta de gestión estratégica en el contexto de las empresas públicas, como lo es el caso específico de la EPULEAM.

En la siguiente tabla 1, se observan los elementos resultantes (categorías y descriptores) de la entrevista en profundidad realizada al Gerente General de la Empresa Pública ULEAM, Economista Ricardo Franco, quien para los efectos de la investigación representa un actor de cambio importante en el proceso de incorporación de estrategias 0 herramientas de gestión dentro de esta empresa pública; y a la Ingeniera Ginger Pincay, Jefe de Talento Humano de la EPULEAM, cuya contribución se da desde la perspectiva de experta de campo, cuyos conocimientos del personal que labora en la empresa permite conocer su visión estratégica y 
operativa sobre el comportamiento organizacional de sus supervisados; así como también la experiencia que aporta en la temática abordada.

Tabla 1. Categorías y Descriptores Emergentes de la Entrevista en Profundidad.

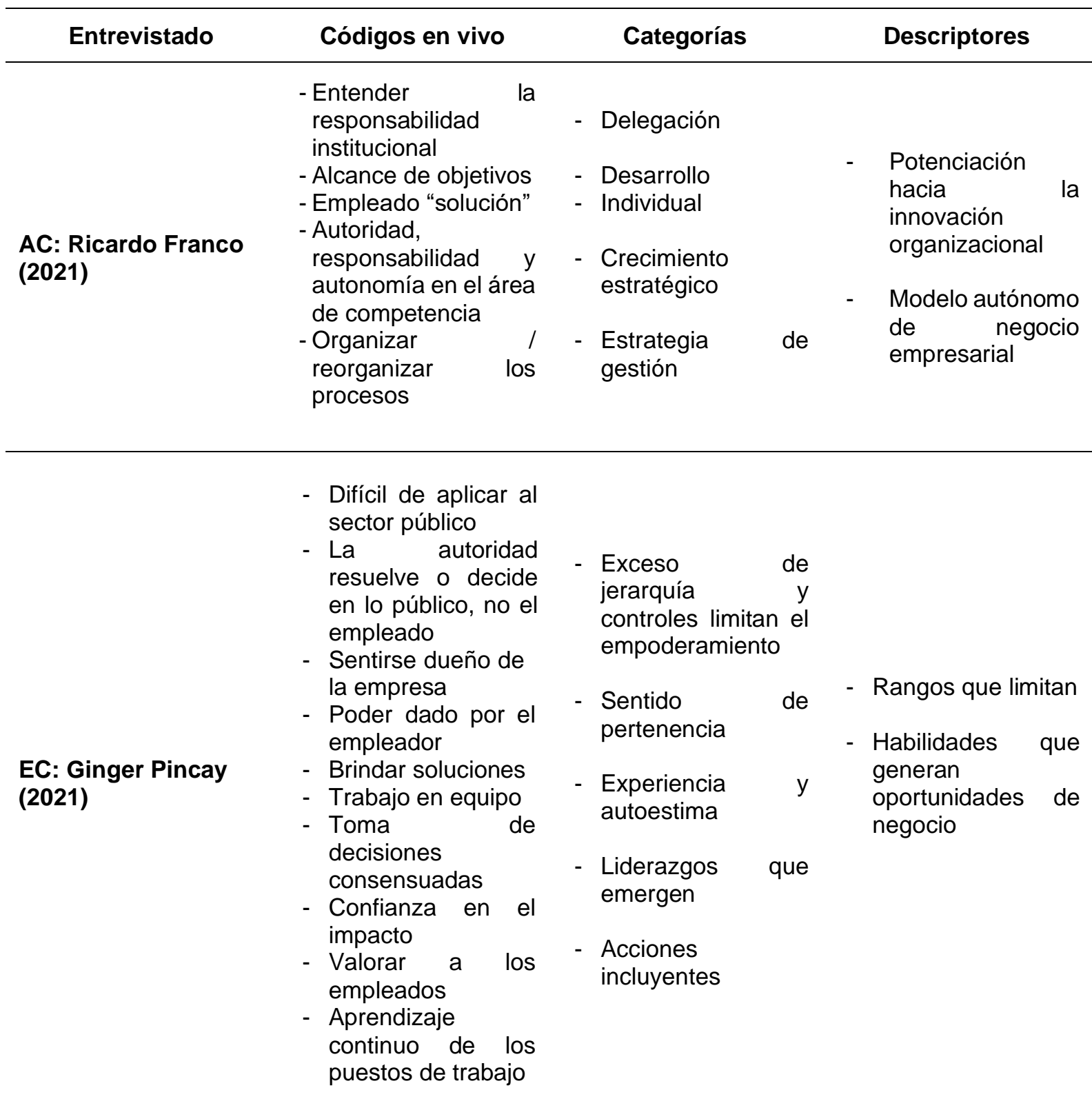

Fuente: Piguave \& Vegas (2021) a partir de entrevistas en profundidad. Notas: AC: Actor de cambio; EC: Experto de campo.

La generación de "códigos in vivo", la triangulación sucesiva entre posturas (referentes teóricos, actores, investigadores), y la posterior consideración de procedimiento de análisis de significados alternativos, originaron una postura sustantiva por parte de los investigadores con relación al empowerment como herramienta de 
gestión estratégica en el contexto de la empresa pública ULEAM; la cual se muestra a continuación a través de una discusión que busca abrir espacios para el debate y la construcción de un nuevo contexto. Veamos.

\section{- Potenciación hacia la innovación} organizacional. Es relevante la necesidad de innovar no solo en la oferta de productos o servicios, sino que esta venga acompañada a su vez de una cultura y comportamiento organizacional basada en la gestión del empowerment empresarial. La innovación debe ser parte de la cultura organizacional y su potenciación se da -en la mayor parte de las veces- desde las bases del capital intelectual de la propia organización. Las empresas públicas representan una oportunidad para los cambios organizacionales en las que se pueda incorporar herramientas empresariales, antes solo exclusivas en las empresas tradicionales o con fines de lucro.

\section{- Modelo autónomo de negocio} empresarial. Los modelos de negocio empresarial tienden a ser autónomos cuando resaltan la peculiaridad de su razón de ser y de su comportamiento frente al mercado. Las empresas públicas deben conceptualizarse desde la base de la autonomía para así poder promover e incursionar en mercados que muchas veces le son ajenos dada su rigidez a la hora de tomar decisiones. Se hace necesario recontextualizar la idea del modelo actual de las empresas públicas, si realmente se quiere a un personal más comprometido, audaz y con sentido de pertenencia.

- Rangos que limitan. Las limitaciones siempre estarán presentes en cualquier tipo de organización; sin embargo, las organizaciones se transforman y buscan los espacios para superar las limitaciones. El empowerment es una herramienta de gestión empresarial que visibiliza la acción de la organización desde la perspectiva individual de cada uno de sus miembros, permitiendo superar las barreras de la autoridad rígida y de las acciones controladas por normas coercitivas, en las que muchas veces solo logran impedir el desarrollo de espacios creativos y con posibilidades de crecimiento organizacional e innovador que realmente conlleven a una 
realización personal y profesional del empleado.

\section{- Habilidades que generan oportunidades de negocio. El} empowerment permite el desarrollo de habilidades gerenciales y laborales vinculadas a la filosofía de la organización y en especial a su cultura de valores institucionales o empresariales. La posibilidad de generar oportunidades de negocio desde la práctica del empowerment es sin dudas, infinita; el empleado o colaborador comienza a observar situaciones, aspectos o espacios que seguramente no han sido explorados 0 explotados en el mercado, llevándolo a proponer estrategias de abordaje $y$ de acciones precisas para el logro del nuevo espacio. Esto sin dudas, se hacen no solo con la confianza que se deposita en el colaborador, sino que se debe agregar la libertad de "pensar de manera creativa y estratégica", así como la incorporación de equipos de trabajo que permitan participar en la construcción de decisiones consensuadas. Desde las empresas públicas, se pueden colocar en práctica estas características y otras que sustentan al empowerment como una herramienta ideal para el fortalecimiento de las habilidades que generan oportunidades de negocio.

Los mercados cambian, por ende, las organizaciones que buscan formar parte de el con sus productos también lo hacen ; por lo cual, desde este espacio de discusión reflexiva, se puede asumir que los descriptores expuestos y definidos anteriormente representan el surgimiento de variables que se deben de considerar en las empresas públicas a fin de que puedan superar los obstáculos impuestos por la rigidez de la autoridad y el marcado control que imponen otras instancias reguladoras, las cuales juegan un papel importante en el desempeño actual de estas empresas públicas.

\section{Desde el espacio conclusivo}

La investigación abre un espacio para exponer sus ideas conclusivas. Estas se focalizan desde los objetivos propuestos al inicio de este artículo, en las mismas se busca resaltar el significado que tiene el empowerment como herramienta de gestión estratégica de los equipos de 
trabajo en el desempeño laboral. Veamos a continuación:

- Es pertinente identificar estrategias de gestión en equipos de trabajo las cuales permitan coadyuvar con el direccionamiento estratégico de los equipos de trabajo para un alto desempeño laboral; la investigación asume que la delegación, las acciones incluyentes, la motivación oportuna y el "dejar ser" contribuyen de manera significativa para que los individuos puedan tener un desarrollo acorde con los valores institucionales y organizacionales, garantizando el emerger de liderazgos transformadores y con espíritu de innovación constante.

- Entender al empowerment como generador de nuevas habilidades en equipos de trabajo frente a las oportunidades de negocio en las empresas públicas es actuar como estratega observador del futuro inmediato del mercado y sus necesidades. Para ello, es conveniente ajustarse a tales necesidades; reinventarse como estructura pareciera ser la opción adecuada, lógicamente amerita compromiso, experiencia, toma de decisiones oportunas, y lo mejor aún, contar con un personal con alto sentido de pertenencia gracias no solo a las recompensas laborales que pueda recibir, sino que se siente parte de un equipo que busca espacios para el crecimiento institucional, la consolidación de proyectos y la construcción de nuevos mercados que permitan a la empresa sostenerse en el tiempo.

- Fortalecer el empowerment pasa por entender los roles individuales y sus principales fortalezas. Sin dudas, es imprescindible la confianza en el impacto de las acciones incluyentes, cuyo objetivo es hacer sentirse responsable de las decisiones que se toman; así como la valoración de los equipos de trabajo de manera integral y sistémica, donde todo depende de todos.

Las empresas públicas, en especial la unidad en estudio EPULEAM, pueden considerar las ideas expuestas en este trabajo investigativo, en la que, con ello pueden lograr abrir espacios para el empowerment organizacional, seguramente la gerencia podrá observar en el corto plazo, como mejoran las tomas de decisiones en cuanto a oportunidad y asertividad, la comunicación se hace más efectiva y el liderazgo se convierte 
en trasformador, además de que la innovación se asume como parte de la filosofía empresarial.

\section{Bibliografía}

Amezcua, E., Pérez, V., y Quiroz, E. (2019). El empowerment como estrategia de crecimiento del talento humano.

Ciencia administrativa, (1), 33-37.

Chiavenato, I. (2009). Administración de Recursos Humanos. Mc Graw Hill Interamericana.

Chiavola, C., Cendrós, P., y Sánchez, D. (2008). El empoderamiento desde una perspectiva del sistema educativo. Omnia, 14(3), 2008, 130-143.

Ivancevich, J., Konopaske, R., y Matteson, M. (2005) Comportamiento organizacional. Mc Graw Hill Interamericana.

Porret, M. (2014). Gestión de personas Manual para la gestión del capital humano en las organizaciones. Esic Editorial.

Cucchi, D. (2008). Empowerment y delegación. En Rabouin, R. (Coord.) Habilidades directivas para un nuevo management. (págs. 55-84). Prentice Hall - Pearson Education
Franco R. (2021). Empowerment como herramienta de gestión estratégica efectiva en el desempeño laboral en equipos de trabajo. Entrevista en profundidad.

Pincay G. (2021). Empowerment como herramienta de gestión estratégica efectiva en el desempeño laboral en equipos de trabajo. Entrevista en profundidad.

Rappaport, J. (1984). Studies in empowerment: Introduction to the issue. Prevention in Human Services.

Strauss, A. y Corbin, J. (2016). Bases de la investigación cualitativa. Técnicas y procedimientos para desarrollar la teoría fundamentada. CONTUSEditorial, Universidad de Antioquia.

Vegas, H. (2016). La teoría fundamentada como herramienta metodológica para el estudio de la gestión pública local. Revista Venezolana de Gerencia., [En línea]

https://produccioncientificaluz .org/index.php/rvg/article/view /21891/21636

Wilson, T. (2000). The Empowerment Manual. Ediciones Gestión. 